\title{
Developing Co-Curricular Activities And Extra-Curricular Activities For All-Round Development Of The Undergraduate Students: A Study Of A Selected Public University In Bangladesh
}

\author{
Md. Roknuzzaman Siddiky \\ Department of Sociology \\ Noakhali Science and Technology University
}

\begin{abstract}
The paper mainly attempts to examine whether co-curricular activities and extra-curricular activities could contribute to the all-round development of the undergraduate students and how these activities may be developed to ensure quality education in a public university in Bangladesh. The study was a qualitative study in which multiple case study and document analysis methods were employed. The study found that the undergraduate students have developed a wide range of personal and social skills including communication skill, organizing skill, presentation skill, public speaking skill and analytical skill by taking part in such activities. The CCAs and ECAs have also facilitated brain development, knowledge acquisition, personality development, and civic development of the students. Hence, the study argued that the CCAs and ECAs have diverse effects to promote allround development of the students. In order to explain how these activities could be developed in a public university, the paper identified several clubs and associations and their key functions. Finally, the paper suggested that the Government and the authority concerned should undertake proper measures for organizing diverse CCAs and ECAs properly and thereby ensure quality education and attain SDGs.
\end{abstract}

Keywords: Quality Education, Co-Curricular Activities, Extra-Curricular Activities, All-Round Development, Personality Development.

$$
\text { تخيص }
$$

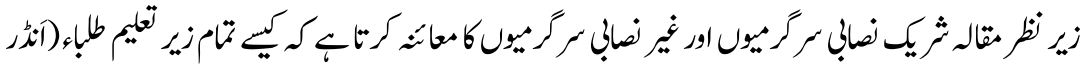

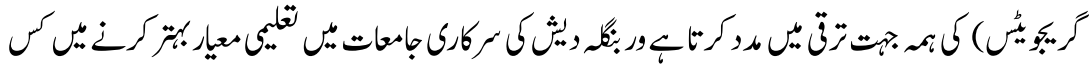

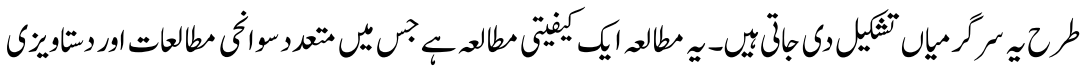

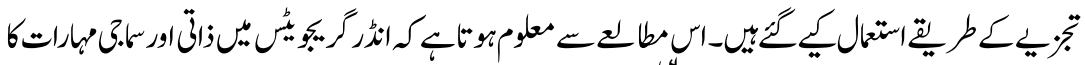

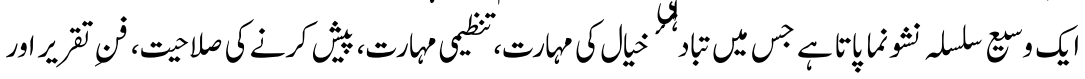

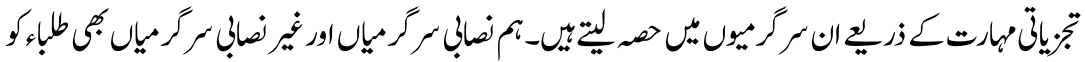

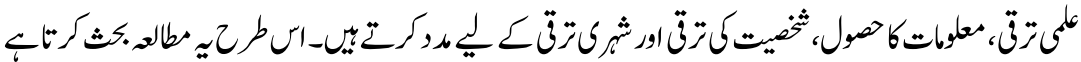

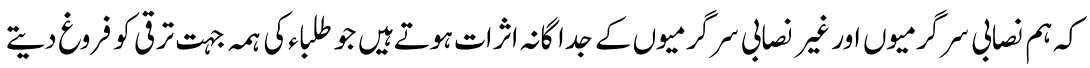




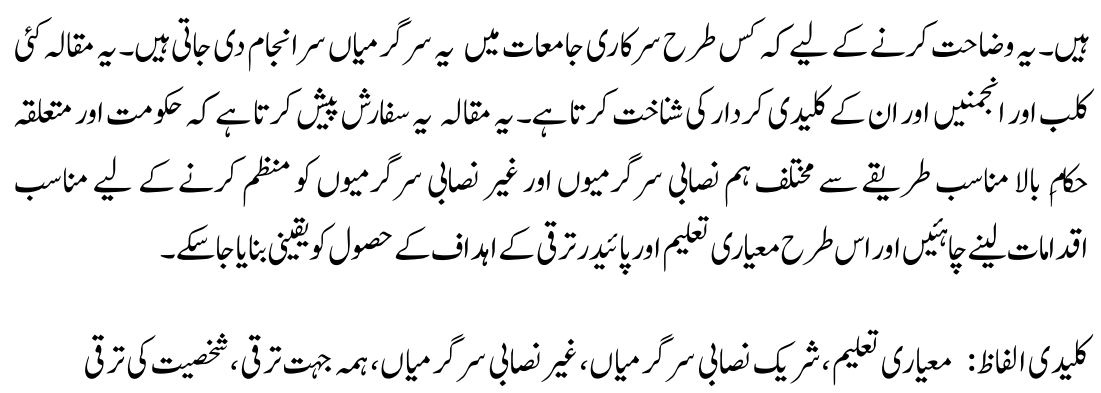

\section{Background of the Study}

Quality education has been defined as one that involves quality learners, quality learning environments, quality content, quality processes and quality outcomes (UNICEF, 2000). Good quality education provides all learners with capabilities they necessitate to become economically productive, develop sustainable livelihoods that would contribute to peaceful and democratic societies and individual well-being (Barrett and Tikly, 2010). Hence, quality education not only involves sound formal academic learning but also includes a wide range of cocurricular activities and extra-curricular activities performed by the students to reach their fullest potential. Ensuring quality education itself is a goal of SDGs (Goal-4) and a means as well for achieving the sustainable development as a whole. It has been regarded not only as an integral part of SDGs but also as a key to promote lifelong learning opportunities for all (Educate A Child, 2016; UNESCO, $2017 \mathrm{a}, 2017 \mathrm{~b})$. As such, it is one of the priority-areas in the SDGs adopted by the international community to foster the development of human capabilities so that they could contribute to the achievement of SDGs in order to make a sustainable, prosperous and equitable planet (UNESCO, 2017a; Lane, 2017). However, to make sure quality education, it is very important to focus on co-curricular activities and extra-curricular activities alongside formal academic learning so that all-round development of the learners can be ensured (Ingale, 2014; Dhanmeher, 2014). In spite of their important role to develop human resources, many public universities of Bangladesh, in particular science and technology based universities are currently in need of building sufficient infrastructure and organizational capacity to develop co-curricular activities and extra-curricular activities. Therefore, one of the major objectives of such universities, that is, to generate skilled manpower in line with global needs and changes in order to contribute to the country's economic and social development, in particular, sustainable development through provision of diverse courses related to science, technology and social science may be hampered. While there is sufficient volume of literature in the academic world about the effects of the CCAs and ECAs on the academic performance and social skill development of the students across the world (Weber, 2008; Wilson, 2009; Daniyal, et al, 2012; Mehmood, et al. 2012; Javed, 2012; Dhanmeher 2014; Ivaniushina and Zapletina, 2015), there has not been so far adequate volume of studies, especially in Bangladesh context as to how the CCAs and ECAs could benefit the students to 
develop their a wide range of personal and social skills and how such activities could be developed within a public university so that their physical, mental, moral, intellectual, and behavioral development, that is, all-round development can be ensured. As such, the paper is intended to investigate the following queries:

a. What sorts of CCAs or ECAs do the students usually like to participate in?

b. How could the students benefit from the CCAs or ECAs?

c. What to be done to develop CCAs or ECAs in a public university in Bangladesh?

\section{Methodology of the Study}

The study is a qualitative study in nature which was deliberately conducted in a science and technology based public university in Bangladesh. Both primary and secondary sources of data were taken into consideration in this study. The study consciously employed multiple case study and document analysis methods to look into the research queries. In order to find out the effects of co-curricular activities or extracurricular activities, secondary data were collected mainly from journal articles and research papers. Primary data were generated from qualitative interviews with a total of 18 purposively selected undergraduate students from randomly chosen 07 academic departments. Out of 18 respondents, 05 were selected from Bangladesh and Liberation War Studies (BLWS) Department, 03 were selected from Pharmacy Department, and 02 were selected from 05 other Departments each namely Statistics, Sociology, Fisheries and Marine Sciences (FIMS), Information and Communication Engineering (ICE) and Applied Mathematics. The respondents were asked a variety of questions about co-curricular activities or extracurricular activities by using open-ended interview-schedule which was designed to find data as to how the respondents could benefit from these activities and what to be done in order to organize such activities effectively so that all-round development could be ensured. The respondents were interviewed indepth by the researcher himself to gain better understanding. Their views were categorized, compared and analyzed very carefully by using tables and underlying themes. Concept-mapping was also used in order to find out the link.

\section{Defining Co-Curricular Activities (CCAs)}

Co-curricular Activities or CCAs (sometimes known as Co-curricular Education) refer to those activities and programs that complement the formal education of the students in educational institution. These activities are usually connected with academic matters and are organized or designed to help the students to have a better understanding of the course, and facilitate their diverse skill development tasks (CSUF, 2010; Ingale, 2014; Dhanmeher, 2014). Mehmood, et al. (2012) suggested that the CCAs are the series of activities related with the school program, which help to bring out all-round development of the students, outside the subjects for examination schedule. However, School District 8, Kootenay Lake (2010) proposed that, 
CCAs refer to any planned excursion away from the school taken by the students, under the direction or supervision of the teacher-in-charge to enrich and extend the classroom instructional program and create links between the school and the community, and to give students practical application of the ideas and theories that they are studying. (p. 1)

Thus the CCAs are such activities that supplement and complement the curricular or main syllabi activities (Leung, $\mathrm{Ng}$ and Chan, 2011). These are very important parts of educational institutions to develop the students' personality, morality, integrity and ethics, and strengthen the classroom learning (Ingale, 2014). The CCAs usually focus on enhancing the students' intellectual capability, mental capability, analytical ability, developing their leadership quality and interpersonal communication skills and allowing them to engage in diverse social networks (Leung, Ng and Chan, 2011; Daniyal et al. 2012; Bartkus et al. 2012; Mehmood, et al. 2012; Dhanmeher, 2014).

\section{Defining Extra-curricular Activities (ECAs)}

Extra-curricular Activities or ECAs (sometimes known as Extra-academic Activities) usually refer to those activities performed by the students that take place outside the regular school curriculum. These activities are generally voluntary and the students do not receive any academic credit or grades out of their participation (Lunenburg, 2010; Singh and Mishra, 2015).

Bartkus et al. (2012) proposed that,

ECAs are defined as academic or non-academic activities that are conducted under the auspices of the school but occur outside of normal classroom time and are not part of the curriculum. Additionally, these sorts of activities do not involve a grade or academic credit and participation is optional on the part of the student. (p. 698)

Hence, ECAs are defined to include those activities which are conducted totally outside the domain of academic curriculum but are essential for the physical, moral, intellectual, behavioral, and civic development of the students. The ECAs allow the students to engage themselves in various social services and nationbuilding activities (Lunenburg, 2010; Bartkus et al. 2012; Le, 2013; Park, 2015; Singh and Mishra, 2015; Freeman, 2017; Chua et al. 2017).

\section{The Scopes of CCAs and ECAs}

CCAs and ECAs are the two words often used interchangeably across the world (Lunenburg, 2010; Leung, Ng and Chan, 2011; Bartkus et al. 2012). At present, there is no clear-cut distinction between these two types of activities since many of such activities are overlapping. Moreover, both the activities aim to enhance the all-round development of the students. However, the CCAs may differ from 
ECAs slightly in terms of nature and scope. Table 1 shows the examples of CCAs and ECAs.

Table: 1

Examples of CCAs and ECAs

\begin{tabular}{|c|c|}
\hline Examples of CCAs & Examples of ECAs \\
\hline 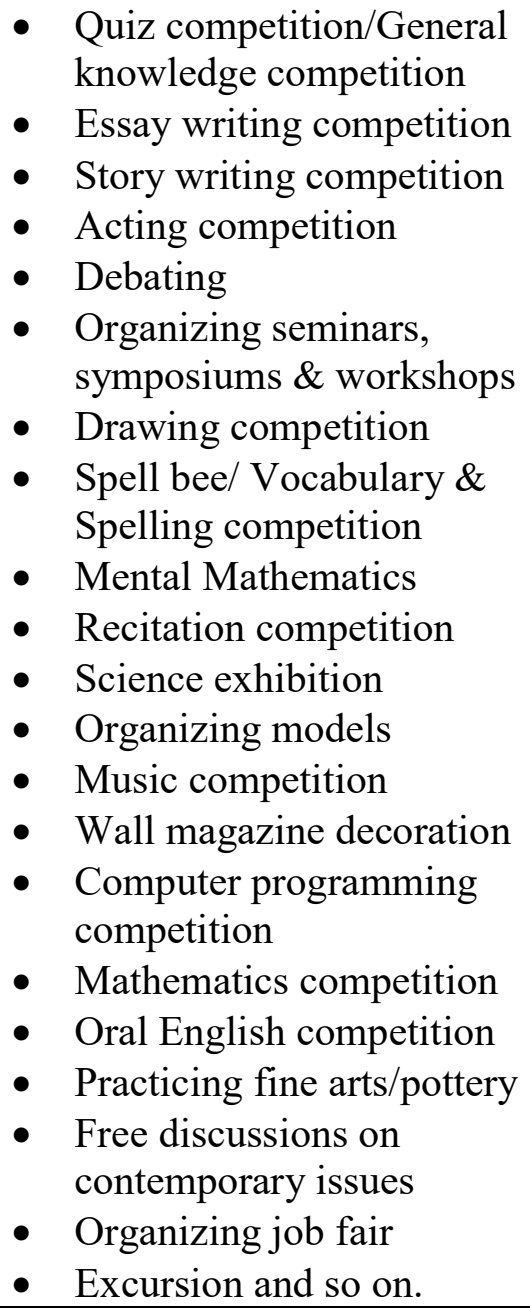 & $\begin{array}{l}\text { - } \text { Athletics/ Gymnastics } \\
\text { - } \text { Cricket tournament } \\
\text { - } \text { Football tournament } \\
\text { - } \text { Swimming competition } \\
\text { - } \quad \text { Table Tennis/Carom Board } \\
\text { - } \text { competition (Indoor Games) } \\
\text { - } \text { Cleanliness program } \\
\text { - } \text { Environmental awareness programs } \\
\text { - } \text { Blood donation program } \\
\text { - Organizing various cultural events } \\
\text { - } \text { Photography (it may be also a co- } \\
\text { curricular activity) } \\
\text { - Tree plantation program } \\
\text { - Teaching street beggars/ elderly } \\
\text { - } \text { Veople } \\
\text { - } \text { Services conducted by the students } \\
\text { Observing various National Days } \\
\text { \& International Days } \\
\text { Observing various cultural } \\
\text { events/days with festivity, and so } \\
\text { on. }\end{array}$ \\
\hline
\end{tabular}

The CCAs are defined as those activities that enhance and enrich the regular curriculum during the normal school day while the ECAs are defined as those activities that broaden the educational experience which usually take place beyond the normal school day (Cumberland Public Schools, 2004). The CCAs are those activities that fall outside the regular academic curriculum and enhance and enrich the curriculum during the normal school day as part of the student's yearly schedule. These activities are mostly faculty-organized and faculty-directed. In contrary, the ECAs are those activities that are extra or additional to the curriculum and may be more leisure-oriented than learning-oriented. These 
activities usually directly may not complement academic studies (Ritchie, 2018). However, this is noteworthy that in the academia, both the terms convey the similar meanings and are used interchangeably. This is also to note that most of the schools or universities across the world do not generally differentiate between CCAs and ECAs since these two sorts of activities are of equal value to ensure all-round development of the students.

\section{Review of Literature}

There are a number of literatures in the academic world concerning the effects of CCAs and ECAs on the students' academic performance and their behavioral and personality patterns. It has been found so far that the CCAs and ECAs have strong association with the students' academic performance and their behavioral or personality patterns. Weber (2008) observed that the students who are involved in the CCAs perform better on state assessments than students who do not participate in such activities. Wilson (2009) found that the students who took part in the ECAs have higher GPAs, a decrease in absenteeism and an increased connectedness to the school. Mehmood, et al. (2012) in their study found that the CCAs have significant relationship with developing some selected personality traits involving selfconfidence, honesty, adaptation, sociability, sympathetic attitude, social obligation and sense of responsibility among secondary school students. They also observed that CCAs have stronger impact on developing such personality traits among male secondary school students than female secondary school students. Daniyal et al. (2012) put forward that the CCAs especially athletic and sports improve the academic performance of the students. Jamal (2012) suggested that the participation in the ECAs promotes the interpersonal skills and professional behaviors of the medical students of the King Abdulaziz University at Jeddah. An experimental study conducted by Bashir and Javed (2012) in order to determine whether the CCAs have an impact on academic performance of the secondary school students. Their study revealed that the CCAs could contribute to the academic performance of the students. Johnston (2013) also found that the ECAs participation have a positive effect on student grade point average and increase the likelihood of students to persist through graduation. Le (2013) found that the ECA participation lowers engagement in risky behaviors for females with low-socio-economic status (SES) more than it does for high-SES females, yet among males the SES gradient is almost non-existent.

Dhanmeher (2014) found that the CCAs have stronger relationship with developing a number personality qualities including adaptation, self-confidence, honesty, sociability, sympathetic attitude, social obligation, sense of responsibility, time management and leadership qualities among Junior College Students. Ivaniushina and Zapletina (2015) put forward that the ECAs have a positive impact on the development of personality and interpersonal skills. They found that the ECAs have helped to develop many personal and social skills of Russian students. Each kind of 
the selected ECAs has different effects on the development of the competencies and interpersonal skills of the students. Singh and Mishra (2015) observed that the ECAs, in particular, Yoga, Horse riding, Sport activities, Dance, Music have significant association with the student's performance in the Government and Private Schools. They found that the benefits of participating in the ECAs involved having better grades, having higher standardized test scores and higher educational attainment, attending school more regularly, and having a higher self concept.

Ismail et al. (2016) observed that the ECAs have a positive impact on the development of personality traits of the students of different universities in Pakistan. Villalobos et al. (2016) found that the CCAs have helped to develop the relationship skills of the Pilipino financial and management accounting students, learn to manage their time effectively and enhance their academic performance. Chua et al. (2017) observed that there is a significant relationship between and among independent variables of number of jobs, number of initial interview, level of participation and soft skills developed through ECAs. Freeman (2017) observed that there is a positive relationship between students' academic performance, in particular GPAs and their involvement in the ECAs. Ritchie (2018) suggested that academic co-curricular activity participation improves Catholic high school students' learning as measured by GPAs. Hence, it may be argued that the CCAs and ECAs have a positive impact not only on the academic performance of the students but also on their social skill development. While CCAs and ECAs may have a positive impact on personal and social skill development of the students, there has not been so far adequate volume of studies as to how these activities influence the all-round development of the undergraduate students in the context of a public university in Bangladesh and what to be done if these sorts of activities are to be organized properly Hence, the study is of great importance not only in Bangladesh context but also in the academia.

\section{Data Analysis and Presentation}

Table: 2

Classification of the respondents

\begin{tabular}{|c|c|c|c|c|c|}
\hline $\begin{array}{c}\text { Respondents' } \\
\text { ID No. }\end{array}$ & $\begin{array}{c}\text { Name of the } \\
\text { Department }\end{array}$ & Year & Term & Age & Sex \\
\hline 1. & Statistics & 2 & 2 & 21 & $\mathrm{M}$ \\
\hline 2. & Statistics & 1 & 2 & 18 & $\mathrm{~F}$ \\
\hline 3. & Sociology & 1 & 2 & 19 & $\mathrm{M}$ \\
\hline 4. & Sociology & 1 & 2 & 19 & $\mathrm{M}$ \\
\hline 5. & BLWS & 1 & 2 & 19 & $\mathrm{~F}$ \\
\hline 6. & BLWS & 1 & 2 & 19 & $\mathrm{M}$ \\
\hline 7. & ICE & 1 & 2 & 19 & $\mathrm{~F}$ \\
\hline 8. & Pharmacy & 1 & 2 & 19 & $\mathrm{~F}$ \\
\hline
\end{tabular}




\begin{tabular}{|c|c|c|c|c|c|}
\hline 9. & BLWS & 1 & 2 & 19 & $F$ \\
\hline 10. & BLWS & 1 & 2 & 19 & $\mathrm{~F}$ \\
\hline 11. & BLWS & 1 & 2 & 19 & $\mathrm{M}$ \\
\hline 12. & Pharmacy & 1 & 2 & 19 & $\mathrm{M}$ \\
\hline 13. & FIMS & 1 & 2 & 19 & $M$ \\
\hline 14. & FIMS & 4 & 2 & 22 & $\mathrm{~F}$ \\
\hline 15. & ICE & 4 & 1 & 22 & $\mathrm{M}$ \\
\hline 16. & $\begin{array}{c}\text { Applied } \\
\text { Math }\end{array}$ & 3 & 1 & 22 & $\mathrm{~F}$ \\
\hline 17. & Pharmacy & 4 & 2 & 24 & $\mathrm{M}$ \\
\hline 18. & $\begin{array}{l}\text { Applied } \\
\text { Math }\end{array}$ & 3 & 2 & 22 & $\mathrm{M}$ \\
\hline
\end{tabular}

Table: 3

Respondents' general views as to CCAs/ECAs

\begin{tabular}{|c|c|c|c|c|c|}
\hline $\begin{array}{l}\text { Respondents' } \\
\text { ID No. }\end{array}$ & $\begin{array}{l}\text { Whether you } \\
\text { are familiar } \\
\text { with } \\
\text { CCAs/ECAs }\end{array}$ & $\begin{array}{c}\text { How do you } \\
\text { come to know } \\
\text { about } \\
\text { CCAs/ECAs? }\end{array}$ & $\begin{array}{l}\text { What sorts of } \\
\text { CCAs/ECAs } \\
\text { you mostly } \\
\text { like? }\end{array}$ & \begin{tabular}{|c|} 
Whether you \\
regularly take part in \\
CCAs/ECAs \\
organized by your \\
university
\end{tabular} & $\begin{array}{c}\text { Whether your } \\
\text { department } \\
\text { organizes } \\
\text { CCAs/ECAs }\end{array}$ \\
\hline 1. & Yes & $\begin{array}{l}\text { From parents, } \\
\text { teachers and } \\
\text { friends }\end{array}$ & $\begin{array}{l}\text { Singing, } \\
\text { dancing }\end{array}$ & Yes & $\begin{array}{c}\text { Occasionally, due } \\
\text { to inadequate } \\
\text { fund }\end{array}$ \\
\hline 2. & Yes & From school & $\begin{array}{l}\text { Various cultural } \\
\text { activities, } \\
\text { indoor sports, } \\
\text { debating }\end{array}$ & Yes & $\begin{array}{l}\text { Occasionally due } \\
\text { to inadequate } \\
\text { fund and tight } \\
\text { semester schedule }\end{array}$ \\
\hline 3. & Yes & From school & $\begin{array}{l}\text { Debating, } \\
\text { sports, cultural } \\
\text { activities. }\end{array}$ & No & $\begin{array}{l}\text { Not always, due } \\
\text { to tight semester } \\
\text { schedule }\end{array}$ \\
\hline 4. & Yes & From school & Sports, debating & Yes & Occasionally \\
\hline 5. & Yes & From school & $\begin{array}{l}\text { Various cultural } \\
\text { activities, } \\
\text { debating, chess }\end{array}$ & Yes & $\begin{array}{c}\text { Occasionally, due } \\
\text { to tight semester } \\
\text { schedule }\end{array}$ \\
\hline 6. & Yes & From college & Debating & Yes & Regularly \\
\hline 7. & Yes & From school & $\begin{array}{c}\text { Debating, poem } \\
\text { recitation }\end{array}$ & Yes & $\begin{array}{l}\text { Occasionally, due } \\
\text { to busy semester } \\
\text { schedule }\end{array}$ \\
\hline 8. & Yes & From school & $\begin{array}{c}\text { Debating, } \\
\text { cultural } \\
\text { activities }\end{array}$ & Yes & $\begin{array}{c}\text { Occasionally, due } \\
\text { to inadequate } \\
\text { fund }\end{array}$ \\
\hline 9. & Yes & From school & $\begin{array}{c}\text { Debating, poem } \\
\text { recitation }\end{array}$ & Yes & Regularly \\
\hline 10 . & Yes & From school & $\begin{array}{c}\text { Debating, } \\
\text { drawing, sports }\end{array}$ & Yes & Regularly \\
\hline 11. & Yes & From college & Debating, music & Yes & Regularly \\
\hline 12. & Yes & From school & Debating, sports & Yes & Regularly \\
\hline
\end{tabular}


Developing Co-Curricular Activities and Extra-Curricular Activities for All-Round Development 69 of the Undergraduate Students: A Study of a Selected Public University in Bangladesh

\begin{tabular}{|c|c|c|c|c|c|}
\hline 13. & Yes & From school & $\begin{array}{c}\text { Social } \\
\text { awareness } \\
\text { building, } \\
\text { debating } \\
\end{array}$ & Yes & $\begin{array}{l}\text { Occasionally, due } \\
\text { to tight semester } \\
\text { schedule }\end{array}$ \\
\hline 14. & Yes & From school & $\begin{array}{l}\text { Acting, music } \\
\text { dancing, sports }\end{array}$ & Yes & $\begin{array}{l}\text { Occasionally due } \\
\text { to tight semester } \\
\text { schedule }\end{array}$ \\
\hline 15. & Yes & From school & $\begin{array}{c}\text { Computer } \\
\text { programming }\end{array}$ & Yes & $\begin{array}{l}\text { Occasionally, due } \\
\text { to busy semester } \\
\text { schedule }\end{array}$ \\
\hline 16. & Yes & From school & $\begin{array}{c}\text { Blood donation, } \\
\text { work for } \\
\text { humanity }\end{array}$ & Yes & $\begin{array}{l}\text { Occasionally, due } \\
\text { to busy semester } \\
\text { schedule }\end{array}$ \\
\hline 17. & Yes & From school & $\begin{array}{c}\text { Social } \\
\text { awareness, } \\
\text { environmental } \\
\text { cleanliness }\end{array}$ & Yes & $\begin{array}{l}\text { Occasionally, due } \\
\text { to busy semester } \\
\text { schedule }\end{array}$ \\
\hline 18. & Yes & From school & $\begin{array}{l}\text { Sports, cultural } \\
\text { activities }\end{array}$ & Yes & $\begin{array}{l}\text { Occasionally, due } \\
\text { to tight semester } \\
\text { schedule }\end{array}$ \\
\hline
\end{tabular}

Table: 4

Respondents' views about the effects/benefits of CCAs/ECAs

\begin{tabular}{|c|c|c|c|c|}
\hline $\begin{array}{l}\text { Respondents' } \\
\text { ID No. }\end{array}$ & $\begin{array}{c}\text { Whether } \\
\text { CCAs/ECAs could } \\
\text { improve your } \\
\text { academic } \\
\text { performance, and } \\
\text { why? }\end{array}$ & $\begin{array}{c}\text { Whether } \\
\text { CCAs/ECAs could } \\
\text { enhance your social } \\
\text { skills/life skills, and } \\
\text { why? }\end{array}$ & $\begin{array}{c}\text { Whether } \\
\text { CCAs/ECAs could } \\
\text { improve your } \\
\text { mental/physical } \\
\text { health, and why? }\end{array}$ & $\begin{array}{l}\text { What sorts of benefits } \\
\text { you have received } \\
\text { from participating in } \\
\text { CCAs/ECAs }\end{array}$ \\
\hline 1. & $\begin{array}{l}\text { No, because these } \\
\text { hamper academic } \\
\text { activities }\end{array}$ & $\begin{array}{l}\text { Yes, because these } \\
\text { enhance speaking } \\
\text { skill }\end{array}$ & $\begin{array}{c}\text { Yes, because these } \\
\text { improve } \\
\text { mental/physical well- } \\
\text { being } \\
\end{array}$ & $\begin{array}{c}\text {-Public speaking skill, - } \\
\text { Program organizing }\end{array}$ \\
\hline 2. & $\begin{array}{l}\text { Yes, because these } \\
\text { improve academic } \\
\text { performance }\end{array}$ & $\begin{array}{l}\text { Yes, because these } \\
\text { help in confidence } \\
\text { building }\end{array}$ & $\begin{array}{l}\text { Yes, because these } \\
\text { help to keep body fit } \\
\text { and mentally } \\
\text { refreshed }\end{array}$ & $\begin{array}{c}\text {-Mental refreshment } \\
\text {-Physical fitness } \\
\text {-Personality } \\
\text { development } \\
\text { - Social interaction }\end{array}$ \\
\hline 3. & $\begin{array}{l}\text { Yes, because these } \\
\text { improve general } \\
\text { knowledge and } \\
\text { language skills }\end{array}$ & $\begin{array}{c}\text { Yes, because these } \\
\text { enhance } \\
\text { interpersonal } \\
\text { communication } \\
\text { skills } \\
\end{array}$ & $\begin{array}{l}\text { Yes, because these } \\
\text { help to keep body fit } \\
\text { and mentally } \\
\text { refreshed }\end{array}$ & $\begin{array}{c}\text {-Extroversion } \\
\text {-Interpersonal } \\
\text { communication skill }\end{array}$ \\
\hline 4. & $\begin{array}{l}\text { Yes, because these } \\
\text { promote brain } \\
\text { development and } \\
\text { intellectual skills }\end{array}$ & $\begin{array}{l}\text { Yes, because these } \\
\text { help in interpersonal } \\
\text { communication and } \\
\text { confidence building }\end{array}$ & $\begin{array}{l}\text { Yes, because these } \\
\text { improve mental and } \\
\text { physical health }\end{array}$ & $\begin{array}{l}\text {-Social Interactions, } \\
\text { - Sociability } \\
\text {-Organizing capability } \\
\text { - Confidence building } \\
\text { - Attentiveness }\end{array}$ \\
\hline 5. & $\begin{array}{l}\text { Yes, because these } \\
\text { help in brain } \\
\text { development and } \\
\text { refreshing }\end{array}$ & $\begin{array}{l}\text { Yes, because these } \\
\text { promote interaction } \\
\text { and connectivity }\end{array}$ & $\begin{array}{l}\text { Yes, because these } \\
\text { help in mental and } \\
\text { physical refreshment }\end{array}$ & $\begin{array}{l}\text { - Extroversion } \\
\text { - Analytical ability } \\
\text { - Planning ability }\end{array}$ \\
\hline 6. & $\begin{array}{l}\text { Yes, because these } \\
\text { increase academic } \\
\text { knowledge and }\end{array}$ & $\begin{array}{l}\text { Yes, because these } \\
\text { increase organizing } \\
\text { skills and public }\end{array}$ & $\begin{array}{l}\text { Yes, because these } \\
\text { help in physical and } \\
\text { mental development }\end{array}$ & $\begin{array}{c}\text {-Organizing skill } \\
\text {-Presentation skill } \\
\text { - Public speaking skill }\end{array}$ \\
\hline
\end{tabular}




\begin{tabular}{|c|c|c|c|c|}
\hline & presentation skills & speaking skills & & \\
\hline 7. & $\begin{array}{l}\text { Yes, because } \\
\text { refreshing mind can } \\
\text { lead to fruitful } \\
\text { learning }\end{array}$ & $\begin{array}{l}\text { Yes, because these } \\
\text { help in public } \\
\text { speaking and } \\
\text { leadership skills }\end{array}$ & $\begin{array}{l}\text { Yes, because these } \\
\text { keep body and mind } \\
\text { fit }\end{array}$ & $\begin{array}{l}\text {-Social Interaction } \\
\text {-Public speaking skill } \\
\text { - Leadership skill }\end{array}$ \\
\hline 8. & $\begin{array}{c}\text { Yes, because these } \\
\text { increase general } \\
\text { knowledge, external } \\
\text { knowledge }\end{array}$ & $\begin{array}{l}\text { Yes, because these } \\
\text { help in interaction } \\
\text { with many people }\end{array}$ & $\begin{array}{l}\text { Yes, because these } \\
\text { revitalize body and } \\
\text { mind }\end{array}$ & $\begin{array}{c}\text {-Public speaking skill } \\
\text {-Presentation skill } \\
\text { - Knowledge } \\
\text { acquisition }\end{array}$ \\
\hline 9. & $\begin{array}{c}\text { Yes, refreshment can } \\
\text { lead to attentive } \\
\text { learning }\end{array}$ & $\begin{array}{l}\text { Yes, because these } \\
\text { enhance public } \\
\text { speaking skills }\end{array}$ & $\begin{array}{l}\text { Yes, because these } \\
\text { revitalize body and } \\
\text { mind }\end{array}$ & $\begin{array}{c}\text {-Social interaction } \\
\text {-Leadership skills } \\
\text {-Public speaking skills }\end{array}$ \\
\hline 10. & $\begin{array}{l}\text { Yes, because these } \\
\text { enrich knowledge } \\
\text { and sharpen IQ }\end{array}$ & $\begin{array}{l}\text { Yes, because these } \\
\text { help in personality } \\
\text { development and } \\
\text { socialization }\end{array}$ & $\begin{array}{c}\text { Yes, because these } \\
\text { enhance physical and } \\
\text { mental fitness }\end{array}$ & $\begin{array}{c}\text {-Physical fitness } \\
\text { - IQ increase } \\
\text {-Socialization }\end{array}$ \\
\hline 11. & $\begin{array}{l}\text { Yes, because these } \\
\text { help in brain } \\
\text { development }\end{array}$ & $\begin{array}{l}\text { Yes, because these } \\
\text { promote interaction } \\
\text { and interpersonal } \\
\text { relationship }\end{array}$ & $\begin{array}{l}\text { Yes, because these } \\
\text { increase mental and } \\
\text { physical refreshment }\end{array}$ & $\begin{array}{l}\text {-Physical and mental } \\
\text { refreshment } \\
\text { - Public speaking skill }\end{array}$ \\
\hline 12. & $\begin{array}{l}\text { Yes, because these } \\
\text { increase refreshment } \\
\text { and attentiveness }\end{array}$ & $\begin{array}{l}\text { Yes, because these } \\
\text { help in knowledge } \\
\text { acquisition and } \\
\text { socialization }\end{array}$ & $\begin{array}{c}\text { Yes, because these } \\
\text { increase physical and } \\
\text { mental refreshment }\end{array}$ & $\begin{array}{c}\text {-Public speaking } \\
\text {-Physical and mental } \\
\text { fitness }\end{array}$ \\
\hline 13. & $\begin{array}{l}\text { Yes, because these } \\
\text { raise attentiveness }\end{array}$ & $\begin{array}{l}\text { Yes, because these } \\
\text { help in networking, } \\
\text { presentation skills }\end{array}$ & $\begin{array}{c}\text { Yes, because these } \\
\text { enhance mental and } \\
\text { physical refreshment } \\
\text { and fitness }\end{array}$ & $\begin{array}{l}\text {-Removing inertia } \\
\text { - Network building } \\
\text {-Presentation skill } \\
\text { - Social responsibility }\end{array}$ \\
\hline 14. & $\begin{array}{l}\text { Yes, because these } \\
\text { help in brain and } \\
\text { analytical } \\
\text { development }\end{array}$ & $\begin{array}{l}\text { Yes, because these } \\
\text { help in network } \\
\text { building and } \\
\text { personality } \\
\text { development }\end{array}$ & $\begin{array}{l}\text { Yes, because these } \\
\text { remove stress and } \\
\text { fatigue }\end{array}$ & $\begin{array}{c}\text {-Removing stress } \\
\text {-Network building } \\
\text {-Personality } \\
\text { development }\end{array}$ \\
\hline 15. & $\begin{array}{l}\text { Yes, because these } \\
\text { complement } \\
\text { academic learning }\end{array}$ & $\begin{array}{l}\text { Yes, because these } \\
\text { help in organizing } \\
\text { capability and team } \\
\text { work building }\end{array}$ & $\begin{array}{l}\text { Yes, because these } \\
\text { enhance physical and } \\
\text { mental refreshment }\end{array}$ & $\begin{array}{l}\text { - Organizing capability } \\
\text { - Problem solving skill } \\
\text {-Mental refreshment }\end{array}$ \\
\hline 16. & $\begin{array}{c}\text { Yes, because these } \\
\text { promote brain } \\
\text { development, a drive } \\
\text { to learn }\end{array}$ & $\begin{array}{l}\text { Yes, because these } \\
\text { help to work for } \\
\text { humanity }\end{array}$ & $\begin{array}{l}\text { Yes, because these } \\
\text { help in physical and } \\
\text { mental refreshment }\end{array}$ & $\begin{array}{l}\text {-To work for humanity } \\
\text { - Communication skill } \\
\text { - Knowledge } \\
\text { acquisition }\end{array}$ \\
\hline 17. & $\begin{array}{c}\text { Yes, because these } \\
\text { raise analytical and } \\
\text { problem-solving } \\
\text { skills }\end{array}$ & $\begin{array}{l}\text { Yes, because these } \\
\text { allow to work for } \\
\text { humanity }\end{array}$ & $\begin{array}{l}\text { Yes, because these } \\
\text { promote physical and } \\
\text { mental refreshment }\end{array}$ & $\begin{array}{l}\text { - Social responsibility } \\
\text {-To work for humanity } \\
\text { - Analytical skill } \\
\text { - Problem solving skill }\end{array}$ \\
\hline 18. & $\begin{array}{l}\text { Yes, because these } \\
\text { promote motivation }\end{array}$ & $\begin{array}{l}\text { Yes, because these } \\
\text { help in team work } \\
\text { building }\end{array}$ & $\begin{array}{l}\text { Yes, because these } \\
\text { help to keep body and } \\
\text { mind energized }\end{array}$ & $\begin{array}{l}\text {-Team work building } \\
\text { - Good social relations } \\
\text { - Organizing skill }\end{array}$ \\
\hline
\end{tabular}


Table: 5

The clubs/associations mostly suggested by the respondents

\begin{tabular}{|c|c|}
\hline $\begin{array}{l}\text { Respondents' } \\
\text { ID No. }\end{array}$ & Clubs/ Associations mostly suggested for CCAs/ECAs \\
\hline 1. & $\begin{array}{l}\text { Model United Nations (MUN) Club, English Language Club, } \\
\text { Computer Club }\end{array}$ \\
\hline 2. & $\begin{array}{l}\text { Debating Club, English Language Club, Indoor Sports Club, } \\
\text { Career Club }\end{array}$ \\
\hline 3. & $\begin{array}{l}\text { Debating Club, Cultural Club, Sports Club, Nature Club, } \\
\text { Rover Scouts }\end{array}$ \\
\hline 4. & $\begin{array}{l}\text { Debating Club, Career Club, Language Club, Sports Club } \\
\text { (Indoor \& Outdoor) }\end{array}$ \\
\hline 5. & $\begin{array}{l}\text { Cultural Club, Literature Club, Debating Society, Rover } \\
\text { Scouts }\end{array}$ \\
\hline 6. & $\begin{array}{l}\text { Debating Club, Cultural Club, Sports Club (Outdoor \& } \\
\text { Indoor), MUN Club }\end{array}$ \\
\hline 7. & $\begin{array}{l}\text { Creative Club, Career Club, English Language Club, Sports } \\
\text { Club }\end{array}$ \\
\hline 8. & $\begin{array}{l}\text { Literature Club, Sports Club, Debating Club, Career Club, } \\
\text { Creative Club }\end{array}$ \\
\hline 9. & $\begin{array}{l}\text { Career Club, Creative Club, English Language Club, Sports } \\
\text { Club }\end{array}$ \\
\hline 10. & $\begin{array}{l}\text { Cultural Club, Debating Club, Hult Prize Competition Club, } \\
\text { Cultural Club }\end{array}$ \\
\hline 11. & $\begin{array}{l}\text { Career Club, MUN Club, Hult Prize Competition Club, } \\
\text { Innovative Club }\end{array}$ \\
\hline 12. & $\begin{array}{l}\text { Debating Club, Career Club, Innovative Club, Cultural Club, } \\
\text { BNCC }\end{array}$ \\
\hline 13. & $\begin{array}{l}\text { Nature, Club, Debating Club, Sports Club, English Language } \\
\text { Club }\end{array}$ \\
\hline 14. & $\begin{array}{l}\text { Career Club, Debating Club, Nature Club, Language Club, } \\
\text { MUN Club }\end{array}$ \\
\hline 15. & $\begin{array}{l}\text { Math Club, Computer Club, Language Club, Debating Club, } \\
\text { Sports Club }\end{array}$ \\
\hline 16. & $\begin{array}{l}\text { Debating Club, Cultural Club, Blood Donation Club, Sports } \\
\text { Club, MUN Club }\end{array}$ \\
\hline 17. & $\begin{array}{l}\text { Sports Club, Environmental Club/ Nature Club, Hult Prize } \\
\text { Competition Club, Career Club, Innovative Club }\end{array}$ \\
\hline 18. & $\begin{array}{l}\text { Cultural Club, Debating Club, Language Club, Sports Club } \\
\text { (Indoor \& Outdoor) }\end{array}$ \\
\hline
\end{tabular}




\section{Research Findings and Discussions}

This is evident from the Table 3 that most of the respondents are familiar with the CCAs or ECAs from their school life. Debating, sports, various cultural activities in particular music and dancing, various social services and awareness building programs are the most common and popular activities among the respondents. Most of the respondents said that they regularly take part in the CCAs or ECAs when the university centrally organizes such activities while their respective departments could not regularly organize such activities due to tight and busy semester schedule, and inadequate funds. Table 4 shows that most of the respondents said that the involvement in CCAs or ECAs could improve academic results or GPA of the students by facilitating their brain development, knowledge acquisition, and raising their analytical skill, attentiveness and mental refreshment. These findings suggested that the participation in CCAs or ECAs could raise the academic performance of the students and hence are consistent with the findings put forward by a number of researchers (Weber, 2008; Wilson, 2009; Daniyal et al., 2012; Javed, 2012; Johnston, 2013; Singh and Mishra, 2015; Freeman, 2017; Ritchie, 2018). However, they did not explain how CCAs and ECAs could increase the academic performance of the students.

The study found that the CCAs or ECAs could improve social or life skill development of the students by raising their interpersonal communication skill, public speaking skill, organizing capabilities, presentation skills as well as by supporting their personality development. The study found that the CCAs or ECAs could improve students' mental and physical health by raising physical and mental refreshment and keeping body and mind sound. The study put forward that most of the respondents themselves have benefited from such activities by raising their public speaking skill, organizing skill, leadership skill, teamwork building skill, communication skill, social interaction and network building skill, presentation skill, and by keeping their physical and mental fitness. The study claimed that the participation in CCAs or ECAs could facilitate the personality development of the students by making them confident, focused, interactive, extrovert and social.

Hence, the study put forward that the participation in CCAs or ECAs have effects on the development of diverse personal and social skills. These findings were supported by the findings presented by many researchers (Jamal, 2012; Mehmood, et al., 2012; Dhanmeher, 2014; Kumar and Selvaraju, 2014; Ivaniushina and Zapletina, 2015; Cariaga and Molina, 2016; Ismail et al., 2016). However, unlike their research findings, the study claimed that involvement in CCAs or ECAs could develop a sense of social responsibility among the students and allow them to work for humanity or society. CCAs or ECAs have also effect on socializing the students. As such, the CCAs or ECAs could facilitate the moral and civic development of the students. The Table 5 shows the clubs/associations mostly suggested by the respondents for 
organizing the CCAs and ECAs include: debating club, sports club (both indoor and outdoor), language club, various cultural clubs, computer club, career club, MUN club, Hult Prize competition club, nature or environmental club, BNCC, Rover Scouts, creative club, innovative club, blood donation club, and so on.

Figure1: Link between the CCAs/ECAs benefits and students' all-round development

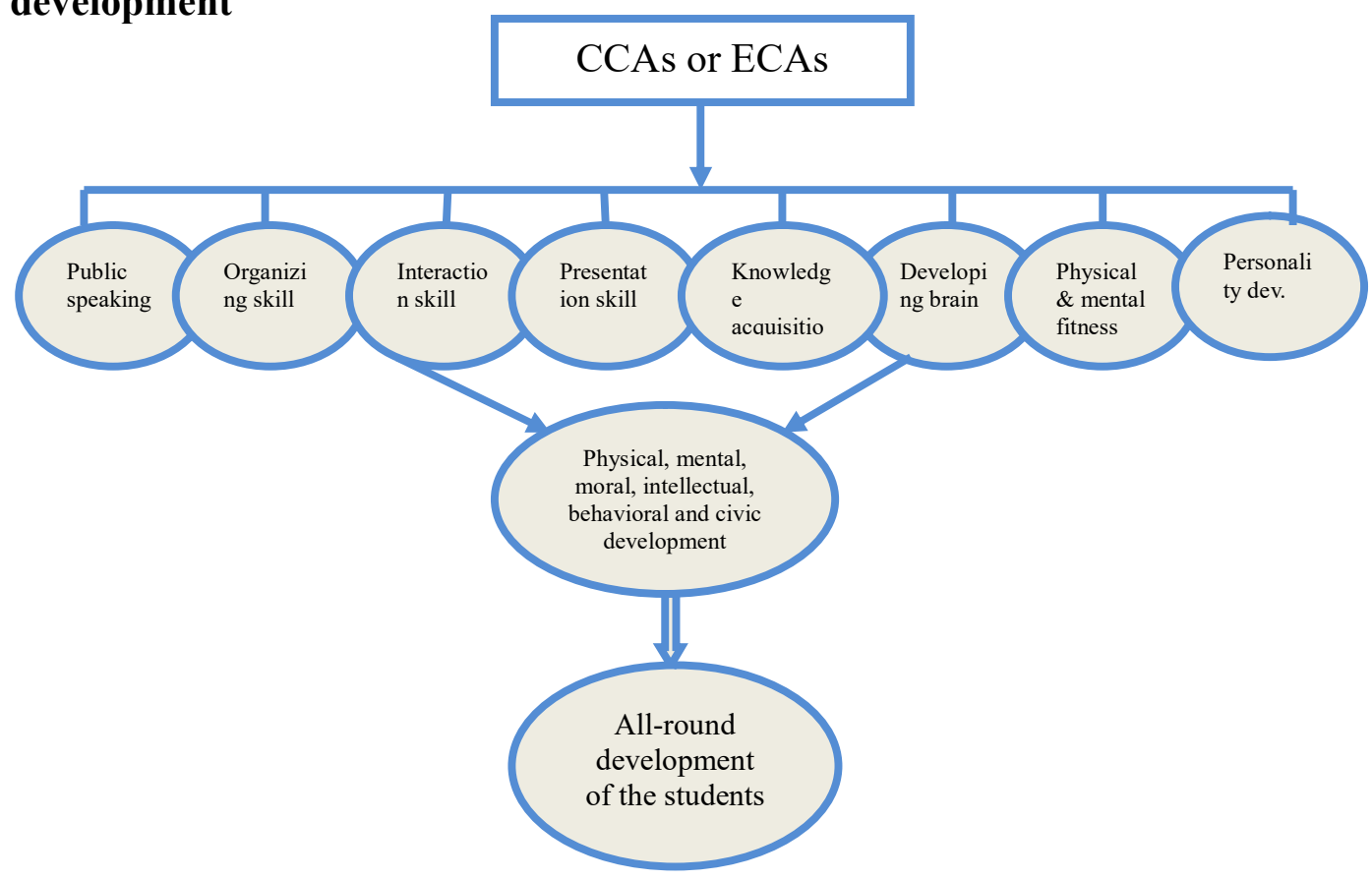

Figure 1 shows the link between the benefits of CCAs or ECAs and the all-round development of the students. As depicted, the CCAs and ECAs have multi-faceted benefits or effects. The empirical findings suggested that the students who regularly take part in the CCAs or ECAs have benefited from such activities in many ways. The CCAs and ECAs have allowed the students to develop many personal and skills such as public speaking skill, social interaction skill, organizing skill and presentation skill. These activities would also facilitate brain development, knowledge acquisition, physical and mental fitness and personality development of the students and thereby promote their physical, mental, moral, intellectual, behavioral and civic development - the all-round development.

\section{Development of CCAs and ECAs}

\section{How to Develop CCAs?}

To organize diverse CCAs in order to ensure all-round development of the students, a public university should preferably set up the following clubs or voluntary associations within its boundary: 


\begin{tabular}{|c|c|c|}
\hline $\begin{array}{l}\text { S. } \\
\text { No. }\end{array}$ & $\begin{array}{c}\text { Name of } \\
\text { Association/ Club }\end{array}$ & Key Activities/Functions \\
\hline 1. & $\begin{array}{l}\text { English } \\
\text { Club/Language Club }\end{array}$ & $\begin{array}{l}\text { - To improve English language proficiency and } \\
\text { other language skills; } \\
\text { - To develop interpersonal communication } \\
\text { skills of the students. }\end{array}$ \\
\hline 2. & Debating Club & $\begin{array}{l}\text { - To develop the students' reasoning power, } \\
\text { analytical ability, democratic attitudes, and } \\
\text { presentation skills. }\end{array}$ \\
\hline 3. & Literature Club & $\begin{array}{l}\text { - To motivate the students to read literature; } \\
\text { - To engage the students in various literary } \\
\text { activities. }\end{array}$ \\
\hline 4. & $\begin{array}{l}\text { Music and Dance } \\
\text { Club }\end{array}$ & $\begin{array}{l}\text { - To engage the students in practicing music } \\
\text { and dance; } \\
\text { - To develop the sense of communal harmony } \\
\text { and humanity among the students. }\end{array}$ \\
\hline 5. & Science Club & $\begin{array}{l}\text { - To motivate the students to practice science, } \\
\text { make scientific models/ project; } \\
\text { - To enhance technological knowledge and } \\
\text { organize science exhibitions. }\end{array}$ \\
\hline 6. & Mathematics Club & $\begin{array}{l}\text { - To motivate the students to learn and practice } \\
\text { mathematics; } \\
\text { - To create a platform to solve various } \\
\text { mathematical problems. }\end{array}$ \\
\hline 7. & $\begin{array}{l}\text { Computer Club/ICT } \\
\text { Club }\end{array}$ & $\begin{array}{l}\text { - To promote the use of computer and its } \\
\text { application in education; } \\
\text { - To encourage the students to engage } \\
\text { themselves in ICT education/ computer } \\
\text { programming. }\end{array}$ \\
\hline 8. & $\begin{array}{l}\text { Innovation Club/ } \\
\text { Creative Club }\end{array}$ & $\begin{array}{l}\text { - To develop the students' creative ideas; } \\
\text { - To engage the students in generating new } \\
\text { ideas } \\
\text { - To engage the students in scientific or } \\
\text { technological innovations. }\end{array}$ \\
\hline 9. & Career Club & $\begin{array}{l}\text { - To provide information about job market; } \\
\text { - To provide career counseling to the students; } \\
\text { - To organize job fair and job-related seminars } \\
\text { etc. }\end{array}$ \\
\hline 10. & Business Club & $\begin{array}{l}\text { - To provide career counseling and job related } \\
\text { information; } \\
\text { - To create the scope for jobs for the Business } \\
\text { Faculty students and introduce them with the } \\
\text { corporate world; }\end{array}$ \\
\hline
\end{tabular}




\begin{tabular}{|c|c|c|}
\hline & & - To build up university-industry relationship. \\
\hline 11. & $\begin{array}{l}\text { Theater/ Drama/ } \\
\text { Fashion Club }\end{array}$ & $\begin{array}{l}\text { - To encourage the students to practice theater } \\
\text { and other various performing arts and thereby } \\
\text { develop their sound personality and increase } \\
\text { their creativity. }\end{array}$ \\
\hline 12. & $\begin{array}{l}\text { BNCC/Rover } \\
\text { Scouts/Rangers etc. }\end{array}$ & $\begin{array}{l}\text { - To develop good moral character and } \\
\text { leadership quality among the students and } \\
\text { engage them in various social services. }\end{array}$ \\
\hline 13. & Red Crescent & $\begin{array}{l}\text { - To engage the students to serve the humanity; } \\
\text { - To prevent and alleviate human suffering } \\
\text { during conflict/natural calamities. }\end{array}$ \\
\hline 14. & $\begin{array}{l}\text { Fine Arts } \\
\text { Club/Center }\end{array}$ & $\begin{array}{l}\text { - To attract the students to learn and practice } \\
\text { drawing/ painting/ pottery/ crafts and other } \\
\text { visual arts. }\end{array}$ \\
\hline 15. & $\begin{array}{l}\text { Model United } \\
\text { Nations Club (MUN } \\
\text { Club) }\end{array}$ & $\begin{array}{l}\text { - To encourage the students to participate in } \\
\text { discussions, debating, and public speaking in } \\
\text { line with UN core values; } \\
\text { - To motivate the students to develop their } \\
\text { leadership quality. }\end{array}$ \\
\hline 16. & $\begin{array}{l}\text { Olympiad Club } \\
\text { (Science) }\end{array}$ & $\begin{array}{l}\text { - To develop the students' interests in Science; } \\
\text { - To create a platform where the students and } \\
\text { teachers will interact to solve various problems } \\
\text { on Science; } \\
\text { - To organize various events/competitions } \\
\text { related to Science. }\end{array}$ \\
\hline 17. & $\begin{array}{l}\text { Hult Prize } \\
\text { Competition Club }\end{array}$ & $\begin{array}{l}\text { - To develop an innovative idea for sound } \\
\text { business initiatives; } \\
\text { - To resolve diverse social problems through } \\
\text { creating innovative ideas/concepts. }\end{array}$ \\
\hline
\end{tabular}

\section{How to Develop ECAs?}

A public university should have the following clubs or voluntary associations to develop a wide range of ECAs so that the physical, mental moral and behavioral development of the students could be ensured:

\begin{tabular}{|c|l|l|}
\hline $\begin{array}{c}\text { S. } \\
\text { No. }\end{array}$ & $\begin{array}{c}\text { Name of } \\
\text { Association/ Club }\end{array}$ & \multicolumn{1}{c|}{ Key Activities/Functions } \\
\hline 1. & Football Club & $\begin{array}{l}\text { - To instruct basic skills of playing football; } \\
\text { - To organize football matches/ competitions; } \\
\text { - To ensure physical and mental fitness of the } \\
\text { students; } \\
\text { - To raise teamwork building among the students. }\end{array}$ \\
& &
\end{tabular}




\begin{tabular}{|c|c|c|}
\hline 2. & Cricket Club & $\begin{array}{l}\text { - To encourage wider interest and involvement in } \\
\text { cricket among the students; } \\
\text { - To offer cricket coaching; } \\
\text { - To organize cricket matches/ competitions; } \\
\text { - To ensure physical and mental fitness of the } \\
\text { students; } \\
\text { - To raise teamwork building among the students. }\end{array}$ \\
\hline 3. & Chess Club & $\begin{array}{l}\text { - To make chess attractive to the students and } \\
\text { thereby improve their thinking ability, strategic } \\
\text { thinking, patience, planning ability, critical } \\
\text { analysis, decision-making ability, and so on. }\end{array}$ \\
\hline 4. & $\begin{array}{l}\text { Athletics } \\
\text { Club/Gymnasium }\end{array}$ & $\begin{array}{l}\text { - To engage the students in physical training and } \\
\text { exercise; } \\
\text { - To keep body and mind fit. }\end{array}$ \\
\hline 5. & $\begin{array}{l}\text { Blood Donation } \\
\text { Club } \\
\text { (e.g., Badhon) }\end{array}$ & $\begin{array}{l}\text { - To encourage the students to donate blood and } \\
\text { thereby allow them to work for humanity; } \\
\text { - To develop a sense of social responsibility. }\end{array}$ \\
\hline 6. & $\begin{array}{l}\text { Environmental } \\
\text { Club/Nature Club }\end{array}$ & $\begin{array}{l}\text { - To create environmental awareness; } \\
\text {-To encourage the students to preserve the } \\
\text { nature, plants, eco-system and biological } \\
\text { diversity etc; } \\
\text { - To organize seminars on environmental issues. }\end{array}$ \\
\hline 7. & Photography Club & $\begin{array}{l}\text { - To train the students about the basic techniques } \\
\text { of photography; } \\
\text { - To organize photo exhibitions on diverse social } \\
\text { issues. }\end{array}$ \\
\hline 8. & $\begin{array}{l}\text { Film Club/ } \\
\text { Society }\end{array}$ & $\begin{array}{l}\text { - To show various short films, art films and } \\
\text { documentary films to the students and thereby } \\
\text { enrich their knowledge. }\end{array}$ \\
\hline 9. & Rotaract Club & $\begin{array}{l}\text { - To allow the students to interact with various } \\
\text { business and professional leaders to promote } \\
\text { humanitarian activities and peace in society; } \\
\text { - To build international liaison to work for } \\
\text { people. }\end{array}$ \\
\hline 10. & $\begin{array}{l}\text { Martial Arts/ } \\
\text { Judo/ Taekwondo } \\
\text { Club }\end{array}$ & $\begin{array}{l}\text { - To popularize martial arts/ Judo/taekwondo } \\
\text { among the students and thereby help them to } \\
\text { maintain good physique and learn self-defense } \\
\text { techniques; } \\
\text { - To raise self-confidence building among the } \\
\text { students. }\end{array}$ \\
\hline 11. & $\begin{array}{l}\text { Swimming } \\
\text { Pool/Club }\end{array}$ & $\begin{array}{l}\text { - To encourage the students to learn and practice } \\
\text { swimming and thereby save life. }\end{array}$ \\
\hline 12. & Badminton & - To promote interaction among the students who \\
\hline
\end{tabular}


Developing Co-Curricular Activities and Extra-Curricular Activities for All-Round Development 77 of the Undergraduate Students: A Study of a Selected Public University in Bangladesh

\begin{tabular}{|l|l|l|}
\hline & $\begin{array}{l}\text { Volleyball } \\
\text { Club/Hadodo Club }\end{array}$ & $\begin{array}{l}\text { have interests in badminton/Volleyball/Hadodo; } \\
- \text { To organize Badminton/Volleyball/Hadodo } \\
\text { competitions. }\end{array}$ \\
\hline 13. & Journalism Club & $\begin{array}{l}\text { - To encourage the students to learn the basic } \\
\text { elements of journalism: planning articles, } \\
\text { interviewing, writing, and online publication. }\end{array}$ \\
\hline 14. & $\begin{array}{l}\text { Bicycle/ Cycling } \\
\text { Club }\end{array}$ & $\begin{array}{l}\text { - To raise physical and mental refreshment of the } \\
\text { students; } \\
- \text { To encourage the students to ride bicycles as an } \\
\text { environmental friendly means of transportation. }\end{array}$ \\
\hline 15. & Ethics Club & $\begin{array}{l}\text { - To increase ethical awareness and develop } \\
\text { morality and integrity among the students. }\end{array}$ \\
\hline 16. & $\begin{array}{l}\text { Student- } \\
\text { Community }\end{array}$ & $\begin{array}{l}\text { - To create a platform where the students, } \\
\text { community people and the police will work } \\
\text { together to combat crime; } \\
- \text { To promote social awareness about crime; } \\
- \text { To develop a sense of social responsibility } \\
\text { among the students. }\end{array}$ \\
\hline
\end{tabular}

\section{Major Suggestions or Recommendations}

Based on empirical findings, the paper puts forward the following key suggestions in order to develop the CCAs and ECAs in newly established public universities, in particular, science and technology based universities in Bangladesh properly:

- The findings of the study suggested that CCAs and ECAs are not regularly organized by most of the departments of the selected science and technology based university due to busy and tight semester schedule in spite of their intense demand from the students, and vital role to promote their all-round development. Hence, all sorts of CCAs and ECAs should be incorporated into the programs conducted on different national and international days so that academic calendar might not be hampered, and tight schedule could be adjusted;

- The study found that inadequate funding is one of the major problems on the part of the departments for not organizing the CCAs and ECAs regularly. As such, the university authority should allocate adequate funds to all the departments and institutes so that such activities may be organized properly;

- To improve English language proficiency and other language skills of the students, a public university should have a Language Club;

- To promote diverse uses of computer in education and organize computer programming competitions, each and every public university in Bangladesh should have a Computer Club or an ICT Club; 
- To develop the students' reasoning power, analytical ability, democratic attitudes, and presentation skills, each and every public university should establish a Debating Club or a Debating Society;

- "A sound body in a sound mind." Hence, a university must have a wellequipped gymnasium or physical fitness center in a separate house/building in order to encourage the students to take physical exercise/ physical training to keep their body and mind fit;

- A university should have a Chess Club in consideration of its importance to facilitate intellectual and mental development of the students;

- c) There is a close connection between environment and development. If environment is affected, development will be automatically affected. Hence, it is very necessary to set up an Environmental Club or a Nature Club so that environmental awareness and attitudes towards preservation of nature among the students could be ensured;

- In view of the globalized world's needs, it is very necessary to make the students creative and innovative. Hence, a university should have an Innovation Club or a Creative Club in order to facilitate the students to generate new ideas or new concepts and engage them in technological innovations;

- Many students do not know the present trends of job market and they do not have proper idea as to how they can prepare themselves for jobs both in the private and public sectors. Hence, it is very necessary to establish a Career Club or a Business Club in a university so that the students' career counseling can be ensured;

- With regard to career development of the students, it is very essential to organize job fair regularly and build up a strong relationship with the industries. In this regard, this is to note that in Korea each and every reputed university is in partnership with the giant companies like Samsung, LG, Hyundai, POSCO, and so on in order to create a platform where both university and industries work together for the development of practical research and students' learning. Hence, building universityindustry partnership is pivotal;

- Now-a-days, Taekwondo is a very much popular game around the world alongside Martial Arts in order to keep body and mind fit, and know the self-defense mechanisms. Hence, it is recommended to establish a Taekwondo Club/ Martial Arts Club;

- Each and every university should have a Football Club and a Cricket Club in order to encourage wider interest and involvement in football and cricket among the students. In this connection, this is to note that organizing intra-department, inter-department and inter-university football/cricket matches is very important.

- To increase ethical awareness and develop morality and integrity among the students of the university, an Ethics Club should be established; 
- Swimming is a very good exercise, and learning swimming is necessary to save life. Nevertheless, most of the universities of Bangladesh do not have any swimming pool. Hence, a Swimming Pool and a Swimming Club should be established;

- Observing various National Days \& International Days with due respect is pivotal;

- Observing various Cultural Events/ Cultural Days with festivity is necessary in order to develop patriotism, harmony and a sense of belonging among the students;

- To enhance the horizon of knowledge of the students, each and every university should organize regularly seminars, symposiums and workshops on relevant academic matters and contemporary social issues;

- To motivate the students to learn and practice Science/Math and solve related problems, each every university should have a Science Club or a Math Club;

- To develop the students' interests in Science/Math, create a platform where the students and teachers will interact to solve diverse problems on Science and Math and to organize various events and competitions concerning Science and Math, Olympiad Club should be established;

- The empirical findings put forward that to develop innovative ideas and promote public speaking skills, critical thinking, leadership skills and presentation skills among the students, Model United Nations activities and Hult Prize Competitions are very important. Hence Model United Nations Club and Hult Prize Competition Club should be established in the public universities.

\section{Conclusions}

In view of the aforesaid discussion, it may be argued that the CCAs and ECAs are multifaceted and multi-dimensional. They are pivotal to facilitate physical development, mental development, moral development, behavioral development and intellectual development of the undergraduate students. They are also very helpful for their personality and civic development. In so doing, they could promote their all-round development. Moreover, these activities could play an important role to allow the students to work for humanity and provide invaluable social services to the distressed people. The CCAs and ECAs could complement academic learning and unleash human potential. Thus, they could contribute to the country's economic and social development by developing a range of personal and social skills. Hence, it is very necessary to ensure the proper development of these two sorts of activities in order to make sure the quality education and sound academic environment in a university, in particular, science and technology based public university in Bangladesh. As such, the Government should come forward and undertake proper policy to develop the CCAs and ECAs properly and thereby achieve SDGs. 


\section{References}

Barkus, K. R., Nemelka, B., Nemelka, M. \& Gardner, P. (2012). Clarifying the Meaning of the Extracurricular Activity: A Literature Review of Definitions. American Journal of Business Education, vol.5:6, pp.693-704.

Barrett, A.M. \& Tikly, L. (2010) Conceptualizing Education Quality towards an EdQual Framework. EdQual. Retrieved from: https://www.edqual.org/ publications/presentations/tbclio10quality.pdf/at_download/file.pdf

Basher, Z. \& Javed, T. (2012). The Effectiveness of Co-Curricular Activities on Academic Students of Secondary Schools Students in District Abbottabad Pakistan. Developing Country Studies, vol.2:2, pp.53-59.

Chua, C.J. E., Chuatoco, I.A. G., Dela Pena, A. M.C., Jimenez, D.L. F. \& Co, D. A. (2017). The Influence of Participation in Extracurricular Activities to the Employability of Industrial Engineering Graduates of One Private University in the Philippines. The Asia Pacific Journal of Multidisciplinary Research, vol.5:2, pp.163-170.

CSUF. (2010). Student Co-Curricular Activities. California State University Fullerton. Retrieved from: http:/hr.fullerton.edu/documents/professionaldevelopment/ ubi/univleadacademy/StudentCo-CurricularReport2010.pdf

Cumberland Public Schools. (2004). Co-curricular and extracurricular activities. Retrieved from: http://www.cumberlandschools.org/system/files/IGDJ\%20 Interscholastic\%20Sports.pdf

Daniyal, M., Nawaz, T., Hassan, A. \& Mubeen, I. (2012). The Effect of CoCurricular Activities on the Academic Performances of the Students: A Case Study of the Islamia University of Bahawalpur, Pakistan. Bulgarian Journal of Science and Education Policy, vol.6:2, pp.257-272.

Dhanmeher, B. R. (2014). Impact of co Curricular Activities on the Non-Academic Development of Junior College Students. M. Phil Thesis, D Y Patil University. Retrieved from: http://www.dypatil.edu/schools/management/ wp-content/uploads/2015/11/impact-of-co-curricular-activities-on-the-nonacademic-development-of-junior-college-students-bharti-dhanmeher.pdf

Educate, A. Child. (2016). Education and the SDGs. Occasional Paper No. 2. Retrieved from: https://educationaboveall.org/uploads/library/file/2a8e 15847d.pdf

Freeman, R. (2017). The Relationship between Extracurricular Activities and Academic Achievement. PhD Thesis, National College of Education, National Louis University. 
Developing Co-Curricular Activities and Extra-Curricular Activities for All-Round Development 81 of the Undergraduate Students: A Study of a Selected Public University in Bangladesh

Ingale, A. R. (2014). Role of Co-Curricular Activities in Student's Life. Scholarly Research Journal for Humanity Science and \& English Language, vol.1:IV, pp.592-594.

Ismail, M., Nadeem, M., Hussain, M. H., Shaheen, M. A., Shahid, M., Ahmad, R. \& Mehmood, U. (2016). Role of ECA's (Extra-Curricular Activities) in Personality Development. International Journal of Research Studies in Biosciences, vol.4:11, pp.47-56.

Ivaniushina, V. A. \& Zapletina, O.O. (2015). Participation in Extracurricular Activities and Development of Personal and Interpersonal Skills in Adolescents. Journal of Siberian Federal University, vol.8:11, pp.2408-2420.

Jamal, A. A. (2012). Developing Interpersonal Skills and Professional Behaviors through Extracurricular Activities Participation: A Perception of King Abdulaziz University Medical Students. JKAU: Med. Science, vol.19:4, pp.3-24.

Johnston, L. H. (2013). The Effects of Extracurricular Activities on Academic Performance and Retention in the Middle Tennessee State University Horse Science Program. Master's Thesis, The Middle Tennessee State University.

Lane, A. (2017). Open Education and Sustainable Development Goals: Making Change Happen. Journal of Learning for Development, vol.4:3, pp.275-286.

Le, T. (2013). Does Participation in Extracurricular Activities Reduces Risky Behavior? Youth in Focus. Youth in Focus Project Discussion Paper Series, No. 13. The Australian National University.

Leung, C., Ng, C. W. R. \& Chan, P. (2011). Can Co-Curricular Activities Enhance the Learning Effectiveness of Students?: An Application to the SubDegree Students in Hong Kong? International Journal of Teaching and Learning in Higher Education, vol.23:3, pp.329-341.

Lunenburg, F. C. (2010). Extracurricular Activities. Sam Houston State University. Schooling, vol.1:1, pp.1-4.

Mehmood, T., Hussain, T., Khalid, M. \& Azam, R. (2012). Impact of CoCurricular Activities on Personality Development of Secondary School Students. International Journal of Humanities and Social Welfare, vol.2:18, pp.139-145.

Park, D. (2015). The Range of Benefits of Extra-Curricular Activities towards English Language Learners. Master's Thesis, Department of Curriculum, University of Toronto. Retrieved from: https://tspace.library.utoronto.ca/ bitstream/1807/68767/1/Park_Daniel_201506_MT_MTRP.pdf 
Ritchie, G.M. (2018). The Impact of Academic Co-Curricular Activity Participation on Academic Achievement: A Study of Catholic High School Seniors. PhD thesis. Seton Hall University. Retrieved from: https://scholarship.shu.edu/cgi/viewcontent.cgi?referer=https:/www.googl e.com $/ \&$ httpsredir $=1 \&$ article $=3564 \&$ context $=$ dissertations

School District 8 Kootenay Lake (2010). Extra and co-curricular activities. Retrieved from: http://www.sd8.bc.ca/Policy/430ExtraandCoCurricular Activities.pdf

Singh, A. \& Mishra, S. (2015). Extracurricular Activities and Student's Performance in Secondary School of Government and Private Schools. International Journal of Sociology and Anthropology Research, vol.1:1, pp.53-61.

UNESCO. (2017a). Education for Sustainable Goals: Learning Objectives. Paris: Author. Retrieved from: http://unesdoc.unesco.org/images/0024/002474/ 247444e.pdf

UNESCO. (2017b). Unpacking Sustainable Development Goal 4. Paris: Author. Retrieved from: http://unesdoc.unesco.org/images/0024/002463/246300E.pdf

UNICEF. (2000). Defining Quality Education. Working Paper Series, New York.

Villalobos, A. S., Dulce, A.C., Fontilar, L.J., Gutierrez, D.C., Sawali, R.C. \& Almero-Encio, H. (2016). Benefits of Co-Curricular Activities to Academic Performance of Financial and Management Accounting Students. Asia Pacific Journal of Arts and Sciences, vol.3:1, pp.83-93.

Weber, B. (2008). Student Involvement in Co-Curricular Activities and Success on the Kansas Mathematics and Reading Assessment. Faculty of School of Education, Baker University. Retrieved from: https://www.bakeru.edu/ images/pdf/SOE/EdD_Theses/Weber_Bill.pdf

Wilson, N. (2009). Impact on Extracurricular Activities on Students. Master's thesis, University of Wisconsin- Stout. Retrieved from: https://www2.uwstout.edu/content/lib/thesis/2009/2009wilsonn.pdf

Md. Roknuzzaman Siddiky is an Associate Professor and Chairman in the Department of Sociology, Noakhali Science and Technology University (NSTU), Bangladesh. 